several weeks later, using a second tetracycline with a different fluorescence pattern. Changes in bone formation that have occurred between the two sets of labels can be measured.

In this study, 21 postmenopausal women with a low BMD or osteoporosis underwent the new procedure with a single iliac-crest biopsy. Overall, 10 randomly selected women received teriparatide and the remaining 11 served as controls. Patients who received teriparatide had increased bone formation on both cancellous and endocortical surfaces, compared with patients who did not receive this anabolic treatment.

The authors conclude that this quadruplelabeling technique is a convenient method to study the short-term effects of anabolic treatments on bone. They highlight that measurements obtained from a single biopsy do not show the large intersite variation that is typical of comparisons of repeated biopsies.

Original article Lindsay R et al. (2006) A novel tetracycline labeling schedule for longitudinal evaluation of the shortterm effects of anabolic therapy with a single iliac crest bone biopsy: early actions of teriparatide. J Bone Miner Res 21: 366-373

\section{Total thyroidectomy is the optimal surgical treatment for childhood thyroid cancer}

The decision whether to perform conservative or radical initial surgery to treat childhood thyroid cancer is a contentious one. Now, a study by Demidchik et al. has found that total thyroidectomy is the best treatment-a change in practice for the team who, up to 1998, favored thyroid-preserving procedures.

The team retrospectively reviewed follow-up data of children treated for thyroid cancer at the Thyroid Cancer Center in Belarus - a part of eastern Europe affected by the 1986 Chernobyl nuclear power station accident. Between 1985 and 2003, 740 children (461 female) were diagnosed with thyroid cancer under the age of 15 years. Over half (426 patients) underwent total thyroidectomy; the rest underwent thyroid-preserving surgery. The authors evaluated the outcome of these different surgical treatments, and determined the risk factors for disease recurrence.

The 10-year, cancer-specific survival rate across the group was $99.2 \%$ (overall survival
98.8\%). Thyroid cancer recurred in over a quarter of the cohort. The independent risk factors for recurrent nodal disease included young age at diagnosis, node-positive disease, multifocal carcinoma, and absence of neck lymph-node dissection. Lung metastases (seen in almost one-fifth of cases) were strongly associated with a young age at diagnosis, the presence of clinical symptoms (e.g. enlarged neck), and female sex. Radioiodine therapy was highly efficacious: over three-quarters of patients with lung metastases demonstrated a complete response, or a stable partial response, to this treatment. Notably, thyroidremnant removal was necessary in over a quarter of the patients who initially underwent a thyroid-preserving operation.

Original article Demidchik YE et al. (2006) Comprehensive clinical assessment of 740 cases of surgically treated thyroid cancer in children of Belarus. Ann Surg 243: 525-532

\section{BMI influences LH levels via the pituitary in polycystic ovary syndrome}

Polycystic ovary syndrome (PCOS), as defined by hyperandrogenism and oligoamenorrhea, is associated with gonadotropin abnormalities: nearly all women with PCOS have an increased luteinizing hormone:follicle-stimulating hormone (LH:FSH) ratio and three-quarters have raised LH levels, compared with healthy women in the early follicular phase. LH levels are known to be inversely related to $\mathrm{BMI}$ in patients with PCOS, but it is unclear whether the effects of $\mathrm{BMI}$ on LH levels are mediated by the pituitary, hypothalamus, or both.

This single-center study evaluated 24 women with PCOS, who had BMls in the range 18.0$48.1 \mathrm{~kg} / \mathrm{m}^{2}$. Study tests were delayed for women who were ovulating or who had menstruated in the previous week. To assess the effects of BMI at the hypothalamic level, Pagán and colleagues measured LH pulse frequency (a surrogate measure of gonadotropin-releasing hormone [GnRH] pulse frequency) and $\mathrm{LH}$ response to submaximal $\mathrm{GnRH}$-receptor blockade using a low dose of the Nal-Glu GnRH antagonist. To assess the effects at the pituitary, the team measured LH response to $75 \mathrm{ng} / \mathrm{kg} \mathrm{GnRH}$ (the dose that triggers ovulation in women with PCOS). 